hep-th/0204051

PUPT-2029

\title{
A semi-classical limit of the gauge/string correspondence
}

\author{
S. S. Gubser, ${ }^{1}$ I. R. Klebanov ${ }^{2}$ and A. M. Polyakov ${ }^{3}$ \\ Joseph Henry Laboratories, Princeton University, Princeton, NJ 08544, USA
}

\begin{abstract}
A world-sheet sigma model approach is applied to string theories dual to fourdimensional gauge theories, and semi-classical soliton solutions representing highly excited string states are identified which correspond to gauge theory operators with relatively small anomalous dimensions. The simplest class of such states are strings on the leading Regge trajectory, with large spin in $A d S_{5}$. These correspond to operators with many covariant derivatives, whose anomalous dimension grows logarithmically with the space-time spin. In the gauge theory, the logarithmic scaling violations are similar to those found in perturbation theory. Other examples of highly excited string states are also considered.
\end{abstract}

April 2002

\footnotetext{
${ }^{1}$ E-mail: ssgubser@princeton.edu

${ }^{2}$ E-mail: klebanov@feynman.princeton.edu

${ }^{3}$ E-mail: polyakov@princeton.edu
} 


\section{Contents}

1 Introduction $\quad 1$

2 Large R-charge revisited $\quad 5$

3 Leading Regge Trajectory $\quad 6$

4 Other macroscopic string states $\quad 9$

4.1 String Spinning on $S^{5} \ldots \ldots \ldots \ldots$. . . . . . . . . . . . . . 10

4.2 Oscillating String . . . . . . . . . . . . . . . . 11

4.3 Arbitrary conformal dimension . . . . . . . . . . . . . . . . . . . 12

5 Logarithms in the perturbative regime $\quad 12$

$\begin{array}{lll}6 & \text { Discussion } & 15\end{array}$

\section{Introduction}

It is by now well established that gauge theories can be described by strings, representing their flux lines. These strings, however, are unusual. In the case of the pure glue gauge theory they propagate in a five dimensional warped space [1] and, if some other fields are present, even in higher dimensions. The string Lagrangian is that of the non-linear sigma model with the target space coinciding with the one described above. The simplest and best explored case of this gauge/string duality is presented by the Yang-Mills theory with $\mathcal{N}=4$ supersymmetry. Conformal invariance and other symmetries of this theory unambiguously determine the geometry of the warped target space. It was found in $[2,3,4]$, developing some earlier findings of [5], that the desired string theory in this case lives in the space $A d S_{5} \times S^{5}$ and that there is a unique prescription relating physical quantities in the string and gauge pictures. Many more complicated examples have been analyzed since then, confirming the existence of a dual string picture for various gauge theories.

However, until very recently, it is mostly the supergravity limit (corresponding to the one-loop approximation of the sigma model) that has been tested, basically because of the challenges inherent in formulating the appropriate sigma models ${ }^{1}$ and of solving them once they are formulated. It has been recently pointed out in [7] that for gauge

\footnotetext{
${ }^{1}$ See $[6]$ and references therein for the most highly developed approach to this problem.
} 
theory operators with very high bare dimension, such as operators with high spin, R-charge, etc., the supergravity approximation is inadequate even at small curvatures of the AdS (corresponding to large 't Hooft coupling $\lambda=g_{\mathrm{YM}}^{2} N$ ). It is expected that for for certain classes of such operators the dimensions asymptotically become integer spaced, even when $\lambda \gg 1$.

These expectations were confirmed and considerably strengthened in [8] for a special class of operators with high R-charge. It turned out to be possible in this case to approximate the AdS space by the much simpler and exactly solvable pp-wave [9] by taking the Penrose limit [10]. What remained unclear was whether this success is a manifestation of a general mechanism.

In this paper we will argue that the gauge theory states with large quantum numbers are described by certain solitons of the nonlinear sigma model. The reason for this is easy to explain. The world sheet dimensions of the $2 \mathrm{~d}$ conformal field theories are expressed in the radial quantization scheme as eigenvalues of the hamiltonian $H=$ $L_{0}+\overline{L_{0}}$. For the small sigma model coupling, the spectrum of such a hamiltonian contains the usual perturbative oscillations which are effectively described in the target space by the supergravity approximation.

However in some cases (which we discuss below) there are also soliton states with world sheet dimensions $\sim \frac{1}{\alpha}$ where $\alpha=1 / \sqrt{\lambda}$ is the sigma model coupling. Discussion of these "conformal solitons" and their gauge counterparts is the main content of the present paper. There is already a well known application of classical solutions: the calculation of the Wilson loop [11]. For closed loops, the relevant solutions (minimal surfaces in $A d S_{5}$ ) are instanton-like, having a finite action once a divergence associated to point-like charges is regulated. Our solutions, by contrast, are solitons, and they have different physical meaning. They describe operators with high bare dimensions (very long "words" in the terminology of [7]). Similar operators describe asymptotic behavior of the deep inelastic scattering.

An important result, which is responsible for some of the phenomenological successes of perturbative QCD in deep inelastic scattering, is the logarithmic dependence of anomalous dimension on spin for high-spin gauge theory operators [12]. With the (relatively) new techniques of AdS/CFT correspondence in hand [2, 3, 4] (for a review see [13]), we might ask how (and whether) logarithmic scaling violations might arise in a regime where stringy techniques in anti-de Sitter space are well-controlled. On the field theory side, such effects need not be tied to a non-zero beta-function: anomalous dimensions of non-chiral operators can arise even in a superconformal theory like $\mathcal{N}=4$ super-Yang-Mills. On the string theory side, it might seem difficult to make progress, because gauge theory operators which are neither chiral nor descendants of chiral operators are thought to correspond to excited string states rather than super- 
gravity modes. Such states have masses at least of order $1 / \sqrt{\alpha^{\prime}}$, so their dimensions typically grow as $\lambda^{1 / 4}[3,4]$. Full control of the string spectrum in $A d S_{5} \times S^{5}$ and the operator-state map seems to be beyond our grasp at present. However, we will argue that certain features of the string spectrum can be well-understood from a non-linear sigma-model perspective, which bypasses (or at least postpones) the usual difficulty with Ramond-Ramond backgrounds.

We will consider a class of gauge invariant operators with high Lorentz spin $S$. These operators are quite different from those with large R-charge: their presence does not rely on the supersymmetry of the gauge theory. In fact, they are well-known to be present in QCD where they were studied early on in the context of deep inelastic scattering [12]. Analogous operators are present in the $\mathcal{N}=4$ supersymmetric $S U(N)$ gauge theory, for example,

$$
\operatorname{tr} X^{I} \nabla_{\left(\mu_{1}\right.} \cdots \nabla_{\left.\mu_{s}\right)} X^{I}
$$

In free field theory, such an operator would have "twist two:" that is, its dimension is $S+2$, while its space-time spin is $S$. Similar operators play an important role in deep inelastic scattering, where leading weak coupling corrections of the form $\Delta=(S+2)+$ $c_{1} g_{Y M}^{2} N \ln S$ can be probed experimentally. Unlike the operators with large R-charge, such high-spin operators cannot be chiral or 'nearly chiral,' hence their anomalous dimensions would seem to be difficult to control at strong coupling. Nevertheless, for sufficiently large $S$, we present a simple semi-classical method for calculating them. Our approach involves finding a soliton solution of the $\sigma$-model describing type IIB strings on $A d S_{5} \times S^{5}$. Although a solution of such a $\mathrm{RR} \sigma$-model is lacking, finding the solitons describing the high-spin closed strings is a much easier problem.

Consider, for example, closed strings on the leading Regge trajectory, i.e. those with the smallest mass for a given spin $S$. It is well known that an approximate description of such states in flat space is a folded closed string which spins as a rigid rod around its center. To study the gauge theory we simply replace the flat space by the global $A d S_{5}$ metric,

$$
d s^{2}=R^{2}\left(-d t^{2} \cosh ^{2} \rho+d \rho^{2}+\sinh ^{2} \rho d \Omega_{3}^{2}\right),
$$

and consider a spinning folded closed string whose center lies at rest at $\rho=0 .^{2}$ We take the usual large $N$ 't Hooft limit keeping $\lambda=g_{\mathrm{YM}}^{2} N$ fixed, and make $\lambda$ very large so that the curvature of the metric is small. For spin $S \ll \sqrt{\lambda}$ we show that the standard AdS/CFT analysis of operator dimensions [3, 4] applies. For $S \sim \sqrt{\lambda}$, however, this analysis becomes inadequate because the string gets appreciably stretched across $\mathrm{AdS}_{5}$. Identifying the energy in the global coordinates with the conformal dimension in the

\footnotetext{
${ }^{2}$ We are grateful to Juan Maldacena for suggesting this to us. In the $\mathrm{AdS}_{4}$ case such a solution was constructed and studied in [14].
} 
dual gauge theory, we find for $S \gg \sqrt{\lambda}$,

$$
\Delta-S=\frac{\sqrt{\lambda}}{\pi} \ln (S / \sqrt{\lambda})+O\left(S^{0}\right)
$$

The result (3) is interesting because these anomalous dimensions grow logarithmically with spin only in gauge theories [12]. Details of the calculation leading to (3) will be provided in section 3 (the logarithmic growth of $\Delta-S$ on the AdS side is closely related to the $\ln S$ scaling of the proper length of the string for large $S$ ). The result (3) shows that for these states the anomalous dimension is small compared to the bare one:

$$
\frac{\Delta-S}{S} \sim \frac{\ln (S / \sqrt{\lambda})}{S / \sqrt{\lambda}} \ll 1 .
$$

Comparison with the perturbative results motivates the following formula for the leading term in the dimension of a high-spin operator on the leading Regge trajectory:

$$
\Delta-S=f(\lambda) \ln S
$$

where $f(\lambda)=a_{1} \lambda+a_{2} \lambda^{2}+\ldots$ is a certain function of the 't Hooft coupling. Indeed, in many gauge theories (including non-supersymmetric ones!) it has been argued that the leading term in the anomalous dimensions grows as $\ln S$ to all orders in perturbation theory, and probably also non-perturbatively [16]. ${ }^{3}$ The analysis of [16] is based on studying Wilson loops with cusps, and the function $f(\lambda)$ appears in this approach as the "cusp anomalous dimension." Therefore, the formula (5) may be a new point of contact between gauge theory and string theory.

Although we discuss operators with high Lorentz spin in the most detail, we believe that our approach of identifying highly excited gauge theory operators with $\sigma$-model solitons is quite general and can be applied to other kinds of operators. Our techniques may also be generalized to backgrounds describing non-conformal gauge theories.

The organization of the paper is as follows. In section 2, we re-examine the case of large R-charge, obtaining the results of [8] from a different perspective, developing ideas in [7]. In section 3, we use similar methods to treat spinning strings in $A d S_{5}$. In section 4 we study some other examples of highly excited strings. And in section 5 we review the appearance of logarithmic scaling violations in perturbative gauge theory and discuss the relation with the results of section 3 .

\footnotetext{
${ }^{3}$ We are grateful to G. Korchemsky for bringing these papers to our attention, and for helpful comments after the original version of this paper appeared.
} 


\section{Large $\mathrm{R}$-charge revisited}

Our work is largely inspired by the recent important observation that, if the bare dimension of an operator becomes large simultaneously with $\lambda$, then the standard rule that the anomalous dimension blows up as $\lambda^{1 / 4}$ may be violated $[7,8]$. A simple example of such a modification is provided by certain operators whose R-charge $J$ is of order $\sqrt{\lambda}$. In [8] it was shown that such operators are in one-to-one correspondence with all type IIB string states in a RR-charged pp wave background which is a Penrose limit of $A d S_{5} \times S^{5}$ [10]. Exact solvability of string theory in this pp wave background [9], together with the AdS/CFT correspondence, predict that the dimensions of these operators are given by

$$
\Delta=J+\sum_{n=-\infty}^{\infty} N_{n} \sqrt{1+\frac{\lambda n^{2}}{J^{2}}},
$$

where $N_{n}$ is the excitation number of the $n$-th string mode. The operators with $N_{n}=0$ for all $n \neq 0$ are chiral while all the rest are non-chiral. Nevertheless, in the limit $\lambda \rightarrow \infty$, where $J^{2} / \lambda$ is held fixed, it is found that the anomalous dimension becomes negligible compared to the bare dimension, $(\Delta-J) / J \rightarrow 0$.

In this section we will reconsider the case of the large $\mathrm{R}$ charge. We will treat it differently (but with the same results) in order to set the stage for the more complicated cases. The bosonic part of the action describing the $\mathrm{AdS}_{5} \times S_{5}$ sigma model is given by

$$
S=\frac{1}{2 \alpha} \int d^{2} \sigma \sqrt{g}\left(\left(\nabla_{\alpha} n\right)^{2}+\left(\nabla_{\alpha} N\right)^{2}\right)+\ldots
$$

where $n$ is a unit vector describing $S^{5}$ and $N$ is a hyperbolic unit vector describing $A d S_{5}$. The dots stand for the fermionic and RR terms. The sigma model coupling $\alpha$ is related to $\lambda$ as $\alpha=\frac{1}{\sqrt{\lambda}}$. Let us consider the $\mathrm{S}_{5}$ part first. The $\mathrm{R}$ charge $J$ is the angular momentum on $S^{5}$. The standard perturbative formula for the world sheet dimension $\delta$ gives for large $J$

$$
\delta=\frac{1}{2} \alpha J^{2}
$$

As was explained in [7], the AdS part gives the same contribution but with the reversed sign (because of the negative curvature) and thus

$$
\delta=\frac{1}{2} \alpha\left(J^{2}-\Delta^{2}\right)
$$

where $\Delta$ is the space-time dimension. The mass shell condition is given by $\delta=-l+1$, where $l$ is the "level," that is the number of the world sheet derivatives in the corresponding operator. From this we see that there should exist gauge theory operators with $\Delta$ and $J$ of order $1 / \alpha$ but with $\Delta-J$ of order 1 . However, we have to correct 
the above formula by accounting for the terms in the world-sheet dimension containing the powers of $\alpha J \sim 1$, while neglecting the powers of $\alpha$. In order to fix the angular momentum we will consider the hamiltonian in the rotating frame $\widetilde{H}=H-\omega J$ and look for the minimal energy. This procedure is equivalent to considering the classical solution for the $S^{5}$ sigma model

$$
\theta=0, \quad \psi=\omega \tau,
$$

where $\theta$ and $\psi$ are the polar and azimuthal angles on $S^{2}$ (we disregard other angles of $S^{5}$, which later will give some trivial modification of our formulae); we parametrize the world sheet by the variables $\tau$ and $\sigma$. Geometrically this solution represents a closed string collapsed to a point which rotates around the equator. The world sheet energy of the above solution is given by $\frac{1}{2 \alpha} \omega^{2}$ which gives the formula (8) after using the relation $J=\frac{\partial E}{\partial \omega}$. To account for the corrections mentioned above, it is sufficient to consider harmonic oscillations near this solution. The Lagrangian for the $\theta$ oscillations, as seen from (7), has the form

$$
\alpha L=\frac{1}{2}\left[(\nabla \theta)^{2}+\omega^{2} \cos ^{2} \theta\right] \simeq \frac{1}{2}\left[(\nabla \theta)^{2}-\omega^{2} \theta^{2}+\omega^{2}\right] .
$$

From here we derive the oscillator spectrum of anomalous dimensions of the spherical sigma model

$$
\delta=\frac{\alpha}{2} J^{2}+\sum_{n} N_{n} \sqrt{n^{2}+\alpha^{2} J^{2}}
$$

where we used the relation $J=\omega / \alpha$. The $N_{n}$ here is the excitation number of the n-th oscillator. The formula for AdS part is obtained by the change $\alpha$ to $-\alpha$. The on-shell condition

$$
\delta\left(S^{5}\right)+\delta\left(A d S_{5}\right) \approx 0
$$

gives the formula (6), which was previously derived in [8] by the use of the Penrose limit. We see that from the sigma model point of view it corresponds to a harmonic approximation to a relatively trivial classical solution (10). We shall now proceed to the more complicated solutions, describing operators with high spin.

\section{$3 \quad$ Leading Regge Trajectory}

In this section we consider a spinning closed string in $A d S_{5}$ to understand the relation between dimension and spin for leading Regge trajectory closed strings. To carry out this calculation it is convenient to use the global $A d S_{5}$ metric (2), so that the energy is 
identified with the conformal dimension in the dual CFT. The string is at the equator of $S^{3}$ and the azimuthal angle depends on time:

$$
\phi=\omega t .
$$

In the Nambu action for the string we pick a gauge where $\tau=t$ and $\rho$ is a function of $\sigma$. The Lagrangian becomes

$$
L=-4 \frac{R^{2}}{2 \pi \alpha^{\prime}} \int_{0}^{\rho_{0}} d \rho \sqrt{\cosh ^{2} \rho-(\dot{\phi})^{2} \sinh ^{2} \rho}
$$

The maximum radial coordinate is $\rho_{0}$, and the factor of 4 comes since there are four segments of the string stretching from 0 to $\rho_{0}$, which is determined by

$$
\operatorname{coth}^{2} \rho_{0}=\omega^{2} .
$$

The energy and the spin of the string are

$$
\begin{aligned}
& E=4 \frac{R^{2}}{2 \pi \alpha^{\prime}} \int_{0}^{\rho_{0}} d \rho \frac{\cosh ^{2} \rho}{\sqrt{\cosh ^{2} \rho-\omega^{2} \sinh ^{2} \rho}} \\
& S=4 \frac{R^{2}}{2 \pi \alpha^{\prime}} \int_{0}^{\rho_{0}} d \rho \frac{\omega \sinh ^{2} \rho}{\sqrt{\cosh ^{2} \rho-\omega^{2} \sinh ^{2} \rho}}
\end{aligned}
$$

The same expressions can also be derived in the conformal gauge where the world sheet action is

$$
S=\frac{1}{4 \pi \alpha^{\prime}} \int d \tau d \sigma G_{i j} \partial_{\alpha} X^{i} \partial^{\alpha} X^{j} .
$$

Now we need to impose the conditions

$$
\begin{aligned}
& T_{++}=\partial_{+} X^{i} \partial_{+} X^{j} G_{i j}=0 \\
& T_{--}=\partial_{-} X^{i} \partial_{-} X^{j} G_{i j}=0 .
\end{aligned}
$$

Inserting $t=e \tau, \phi=e \omega \tau, \rho=\rho(\sigma)$ into these equations, we find

$$
\left(\rho^{\prime}\right)^{2}=e^{2}\left(\cosh ^{2} \rho-\omega^{2} \sinh ^{2} \rho\right) .
$$

Thus,

$$
d \sigma=\frac{d \rho}{e \sqrt{\cosh ^{2} \rho-\omega^{2} \sinh ^{2} \rho}} .
$$

We may now adjust $e$ so that the period of $\sigma$ is $2 \pi$.

The space-time energy is given by

$$
E=\frac{R^{2}}{2 \pi \alpha^{\prime}} e \int_{0}^{2 \pi} d \sigma \cosh ^{2} \rho,
$$


and the spin by

$$
S=\frac{R^{2}}{2 \pi \alpha^{\prime}} e \omega \int_{0}^{2 \pi} d \sigma \sinh ^{2} \rho .
$$

Changing the integration variable from $\sigma$ to $\rho$ we find the previously derived expressions.

Since $R^{4}=\lambda \alpha^{\prime 2}$, where $\lambda$ is the 't Hooft coupling, these expressions specify $E / \sqrt{\lambda}$ and $S / \sqrt{\lambda}$ as functions of $\omega$. Therefore, the dependence of $E / \sqrt{\lambda}$ on $S / \sqrt{\lambda}$ is known in parametric form. Actually, the integrals in (17) and (18) can be expressed in terms of elliptic or hypergeometric functions, as we shall explain in section 4.3. Here it will suffice to give approximate expressions in the limits where the string is much shorter or much longer than the radius of curvature of $A d S_{5}$.

Short strings. For large $\omega, \rho_{0} \approx 1 / \omega$. Here the string is not stretched much compared to the radius of curvature of $A d S_{5}$, so we can approximate $A d S_{5}$ by flat metric near the center. The calculation reduces to the standard spinning string in flat space, and we get

so that

$$
E=\frac{R^{2}}{\alpha^{\prime} \omega}, \quad S=\frac{R^{2}}{2 \alpha^{\prime} \omega^{2}},
$$

$$
E^{2}=R^{2} \frac{2 S}{\alpha^{\prime}} .
$$

Using the AdS/CFT correspondence, we have $\Delta=E$. For large $\omega, S \ll \sqrt{\lambda}$. In this regime we find agreement with the AdS/CFT result

$$
\Delta^{2} \approx m^{2} R^{2} .
$$

Indeed, for the leading closed string Regge trajectory, $m^{2}=\frac{2(S-2)}{\alpha^{\prime}}$, so the precise factor in (26) agrees with the AdS/CFT formula.

Long strings. The situation where $S \gg \sqrt{\lambda}$ corresponds to $\omega$ approaching 1 from above:

$$
\omega=1+2 \eta,
$$

where $\eta \ll 1$. Then $\rho_{0}$ becomes very large, so that the string feels the metric near the boundary of AdS:

$$
\rho_{0} \rightarrow \frac{1}{2} \ln (1 / \eta) .
$$

Expanding the integrals for $E$ and $S$, one finds

$$
\begin{aligned}
& E=\frac{R^{2}}{2 \pi \alpha^{\prime}}\left(\frac{1}{\eta}+\ln (1 / \eta)+\ldots\right), \\
& S=\frac{R^{2}}{2 \pi \alpha^{\prime}}\left(\frac{1}{\eta}-\ln (1 / \eta)+\ldots\right) .
\end{aligned}
$$




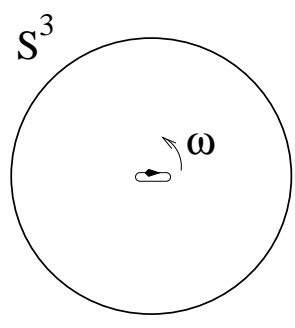

(a)

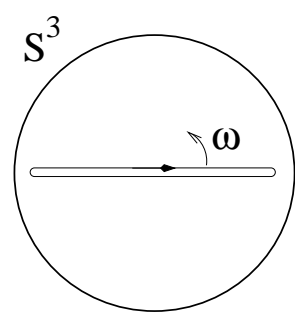

(b)

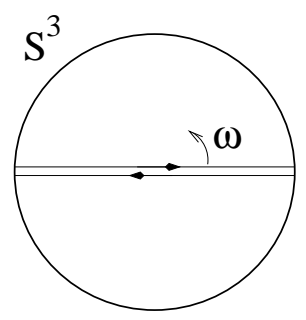

(c)

Figure 1: Short, long, and infinite strings spinning in $A d S_{5}$, shown here in cross-section.

It follows that $E-S$ does not approach a constant in the limit $S \gg \sqrt{\lambda}$, but instead behaves as follows:

$$
E-S=\frac{\sqrt{\lambda}}{\pi} \ln (S / \sqrt{\lambda})+\ldots
$$

Thus, the situation is different from the large R-charge limit: the dependence of the dimension on large spin is more complicated than for large R-charge. The logarithmic asymptotics in (32) are qualitatively the same as in perturbative gauge theories, a topic which we will discuss in section 5 .

It may be necessary to take the string coupling very small in order to suppress decay of the long spinning strings into a number of shorter strings corresponding to operators which are chiral primaries or descendants of them. In the strict ' $t$ Hooft limit, $g_{\mathrm{str}} \rightarrow 0$, hence these states are stable.

Infinite strings: Although energy and angular momentum diverge as $\omega \rightarrow 1$ from above, one may nevertheless consider solutions of the form (14) with $\omega<1$ : such a solution represents two Wilson loops stretching in opposite directions, with the ends rotating on the equator of $S^{3}$ with velocity $\omega$. See figure 1.

\section{Other macroscopic string states}

The approach used in section 3 to treat strings spinning in $A d S_{5}$ can also be used to approximately describe other highly excited string states. In sections 4.1 and 4.2 , we present two examples. In section 4.3, as a formal exercise, we extend the discussion to states in the sigma model which do not necessarily satisfy the on-shell conditions. 


\subsection{String Spinning on $S^{5}$}

A similar calculation may be done for a string whose center of mass is not moving on $S^{5}$, but which spins around that point and is correspondingly stretched. We lose nothing by assuming that this point is the north pole. Let us write the $S^{5}$ metric as

$$
d s^{2}=d \theta^{2}+\sin ^{2} \theta d \phi^{2}+\cos ^{2} \theta d \Omega_{3}^{2},
$$

and adopt the ansatz $t=e \tau, \phi=e \omega \tau, \theta=\theta(\sigma)$. Then from $T_{++}=T_{--}=0$ we get

$$
\left(\theta^{\prime}\right)^{2}=e^{2}\left(1-\omega^{2} \sin ^{2} \theta\right) .
$$

Thus,

$$
d \sigma=\frac{d \theta}{e \sqrt{1-\omega^{2} \sin ^{2} \theta}} .
$$

We may now adjust $e$ so that the period of $\sigma$ is $2 \pi$. The folded closed string is stretched along the $\theta$ direction up to $\theta_{0}$ given by

$$
\sin \theta_{0}=\frac{1}{\omega} .
$$

The space-time energy is given by

$$
E=\frac{R^{2}}{2 \pi \alpha^{\prime}} e \int_{0}^{2 \pi} d \sigma=4 \frac{R^{2}}{2 \pi \alpha^{\prime}} \int_{0}^{\theta_{0}} d \theta\left(1-\omega^{2} \sin ^{2} \theta\right)^{-1 / 2},
$$

and the R-charge by

$$
J=\frac{R^{2}}{2 \pi \alpha^{\prime}} e \omega \int_{0}^{2 \pi} d \sigma \sin ^{2} \theta=4 \frac{R^{2}}{2 \pi \alpha^{\prime}} \omega \int_{0}^{\theta_{0}} d \theta\left(1-\omega^{2} \sin ^{2} \theta\right)^{-1 / 2} \sin ^{2} \theta .
$$

These integrals can be expressed in terms of elliptic or hypergeometric functions, but it would add little to the discussion to present the explicit forms. As before, the interesting regime is when $\omega$ approaches 1 from above, $\omega=1+2 \eta$. As $\eta \rightarrow 0$, $\theta_{0} \rightarrow \pi / 2$. Both $E$ and $J$ diverge as $\ln \eta$ at leading order while their difference stays finite:

$$
E-J \rightarrow \frac{2 R^{2}}{\pi \alpha^{\prime}}=2 \frac{\sqrt{\lambda}}{\pi} .
$$

This is to be contrasted with the string spinning in $A d S_{5}$ : there, $E-S \sim \sqrt{\lambda} \ln S$, while here $E-J \sim \sqrt{\lambda}$. We believe that the logarithm is due to the many gauge-covariant derivatives that carry the spin $S$ in the dual gauge theory.

As for the strings spinning in $A d S_{5}$, it seems likely that the strings spinning in $S^{5}$ can decay into BPS or almost BPS states: physically, this corresponds to the string breaking and descending from the north pole to the equator. Such processes can be 
suppressed by taking $g_{\text {str }} \ll 1$. Also in analogy to strings spinning in $A d S_{5}$, we may continue past the limit $\omega<1$, but the solutions again change character: instead of a string doubled back on itself, and stretched out because of its angular momentum, we must now consider a string stretched around a great circle of $S^{5}$, and rotating so that its velocity at the equator of $S^{5}$ is $\omega$. Such states are unstable toward "slipping off the side" of the $S^{5}$. The relation (34) still holds, and the energy and angular momentum of these great-circle states may be expressed as

$$
\begin{aligned}
& E=4 \frac{R^{2}}{2 \pi \alpha^{\prime}} \int_{0}^{2 \pi} d \theta\left(1-\omega^{2} \sin ^{2} \theta\right)^{-1 / 2} \\
& J=4 \frac{R^{2}}{2 \pi \alpha^{\prime}} \omega \int_{0}^{2 \pi} d \theta\left(1-\omega^{2} \sin ^{2} \theta\right)^{-1 / 2} \sin ^{2} \theta .
\end{aligned}
$$

As usual, the integrals can be done in terms of elliptic or hypergeometric functions.

\subsection{Oscillating String}

Now let us consider a closed string which oscillates around the center of $A d S_{5}$. It first expands from the center of $A d S_{5}$, reaches a maximum $\rho$ and then recontracts back to zero; then this motion repeats. For such a string, $\phi=\sigma$ and $\rho=\rho(\tau)$.

The space-time energy is a constant of motion:

$$
E=\frac{R^{2}}{\alpha^{\prime}} \frac{d t}{d \tau} \cosh ^{2} \rho
$$

From $T_{++}=T_{--}=0$ we find

$$
(\dot{\rho})^{2}+\sinh ^{2} \rho-e^{2} \cosh ^{-2} \rho=0,
$$

where $e=E \alpha^{\prime} / R^{2}$.

The maximum radius of the string is found from

$$
\sinh ^{2}\left(2 \rho_{0}\right)=4 e^{2} .
$$

Presumably these strings correspond to highly excited sigma model operators. It is fairly clear that the excitation level is related to the amplitude $\rho_{0}$. When $E / \sqrt{\lambda}$ is

small we find harmonic oscillations of amplitude $\rho_{0}$. Then $\rho_{0}^{2}$ should be proportional to the excitation level of the oscillation:

$$
\rho_{0}^{2} \sim \frac{n}{\sqrt{\lambda}}
$$

which insures the validity of the usual AdS/CFT relation between $\Delta$ and the level. It is less clear how to extrapolate to large $E / \sqrt{\lambda}$ where the oscillations are no longer small, and it is also mysterious to us precisely what gauge theory operators these states correspond to. 


\subsection{Arbitrary conformal dimension}

As a formal exercise, we can generalize the results of section 3 to solutions that don't obey the constraints, but rather have $T_{++}=T_{--}=\delta-1$ for some arbitrary conformal dimension $\delta$. In this way we hope to get an asymptotic formula of the type $\alpha \delta=$ $f(\alpha \Delta, \alpha S)$, for the spinning string in $A d S_{5}$. Such a formula is an approximate scaling form, good when $\alpha \ll 1$ but $\alpha \delta, \alpha \Delta$, and $\alpha S$ are finite. Equation 32 is recovered by setting $\delta=1$.

From $T_{++}=T_{--}=\delta-1$, we arrive at a slight modification of our previous treatment of the string spinning in $A d S_{5}$ : now

$$
\left(\frac{d \rho}{d \sigma}\right)^{2}=\frac{4(\delta-1)}{R^{2}}+\cosh ^{2} \rho-\omega^{2} \sinh ^{2} \rho,
$$

but the expressions (23) and (24) for the energy and spin are unchanged. The integrals can still be done in terms of hypergeometric functions. The result is best expressed be defining

$$
s=\frac{2 \pi \alpha^{\prime}}{R^{2}} S \quad e=\frac{2 \pi \alpha^{\prime}}{R^{2}} E \quad a=\sqrt{1+4(\delta-1) / R^{2}} .
$$

Then we obtain

$$
\begin{aligned}
& s=\frac{\pi \omega}{\sqrt{\omega^{2}-1}} \frac{a^{2}}{\omega^{2}-1}{ }_{2} F_{1}\left(\frac{1}{2}, \frac{3}{2} ; 2 ; \frac{a^{2}}{\omega^{2}-1}\right) \approx \frac{a}{\eta}+\frac{1}{a} \ln \eta \\
& e=\frac{2 \pi}{\sqrt{\omega^{2}-1}}{ }_{2} F_{1}\left(-\frac{1}{2}, \frac{1}{2} ; 1 ; \frac{a^{2}}{\omega^{2}-1}\right) \approx \frac{a}{\eta}-\frac{1}{a} \ln \eta,
\end{aligned}
$$

where as usual, $\omega=1+2 \eta$. Solving for $a$, one finds

$$
a \approx \frac{2}{e-s} \ln (e+s) .
$$

Trivial algebra leads to an explicit though slightly messy expression for the function $f$ in the asymptotic relation $\alpha \delta=f(\alpha \Delta, \alpha S)$. Setting $\delta=1$ is equivalent to $a=1$, and with this constraint, (48) leads back to (3).

\section{Logarithms in the perturbative regime}

Logarithmic scaling violations are a characteristic feature of perturbative gauge theory in four dimensions, observed, for instance, for gauge theory operators of large dimension but low "twist" - where twist is dimension minus spin. Their anomalous dimensions 
are responsible for violations of the Bjorken scaling in deep inelastic scattering [12]. As an example, consider the operator

$$
\mathcal{O}_{\mu_{1} \ldots \mu_{n}}^{\phi}=\phi^{*} \nabla_{\left(\mu_{1}\right.} \cdots \nabla_{\left.\mu_{n}\right) \mid} \phi
$$

for a gauge theory with some scalar fields transforming in a given representation (which we shall eventually specialize to the adjoint, as appropriate to $\mathcal{N}=4$ supersymmetric Yang-Mills theory). The symmetrization (...)| denotes also a removal of traces. We restrict to even $n$ since this is the only non-zero case for $\mathcal{N}=4 \mathrm{SYM}$. The gaugecovariant derivative is $\nabla_{\mu}=\partial_{\mu}+i g_{Y M} A_{\mu}$. In the following calculation, we will not attempt to construct conformal primaries - so in principle there can be mixing between the operators we consider and others of twist two. Nor will we try to write down the one-loop amplitudes with all factors and signs precise: the main point will be to remind the reader of why the dominant one-loop effects grow logarithmically with $n$. In the free theory, the operator $\mathcal{O}^{\phi}$ has dimension $n+2$ and spin $n$, hence twist 2 . We would like to inquire what anomalous dimension arises at one loop. This is closely analogous to a classic perturbative QCD calculation [12]. The dominant graph has two scalars and a gauge boson coming out of the vertex, and the gauge boson rejoining one scalar line. It is convenient to introduce

$$
\mathcal{O}_{\Delta}^{\phi}=\mathcal{O}_{\mu_{1} \ldots \mu_{n}}^{\phi} \Delta^{\mu_{1}} \cdots \Delta^{\mu_{n}}
$$

for any vector $\Delta^{\mu}$. The anomalous dimension can be read off from the logarithmic term in the amplitude for the dominant graph:

$$
\mathcal{M}=i g_{Y M}^{2} C_{2}(R) \int \frac{d^{4} p}{(2 \pi)^{4}} \frac{1}{p^{2}(p-k)^{2}} \sum_{j=0}^{n-1}(\Delta \cdot p)^{j}(\Delta \cdot k)^{n-j}=c_{\phi}(\Delta \cdot k)^{n} \ln \Lambda^{2}+\ldots
$$

where $C_{2}(R)$ is the Casimir of the representation in which $\phi$ falls. The $j^{\text {th }}$ term in the sum comes from the term

$$
i g_{Y M} \phi^{*}\left(\partial_{\mu_{1}} \cdots \partial_{\mu_{j}} A_{\mu_{j+1}}\right) \partial_{\mu_{j+2}} \cdots \partial_{\mu_{n}} \phi
$$

in $\mathcal{O}_{\mu_{1} \ldots \mu_{n}}^{\phi}$. We still intend the traceless symmetrization of $\mu_{1} \ldots \mu_{n}$, though it is no longer explicit in the notation. To kill off all contact terms and pick up only the logarithmic term that rescales $\mathcal{O}_{\mu_{1} \ldots \mu_{n}}^{\phi}$, we differentiate $n$ times with respect to $k$ and then set $k=0$ :

$$
\begin{aligned}
& i g_{Y M}^{2} C_{2}(R) \int \frac{d^{4} p}{(2 \pi)^{4}} \sum_{j=0}^{n-1}\left(\begin{array}{c}
n \\
j
\end{array}\right) \frac{2^{j} j !}{p^{2 j+4}} p_{\mu_{1}} \cdots p_{\mu_{j}}(\Delta \cdot p)^{j}(n-j) ! \Delta_{\mu_{j+1}} \cdots \Delta_{\mu_{n}} \\
& =c_{\phi} n ! \Delta_{\mu_{1}} \cdots \Delta_{\mu_{n}} \ln \Lambda^{2} .
\end{aligned}
$$


The integral diverges logarithmically. Replacing $\int_{0}^{\infty} \frac{d p}{p}$ by $\ln \Lambda$, we wind up with

$$
\frac{i g_{Y M}^{2} C_{2}(R)}{16 \pi^{2}} \frac{1}{2 \pi^{2}} \int_{S^{3}} d^{3} \Omega \sum_{j=0}^{n-1} 2^{j} \hat{p}_{\mu_{1}} \cdots \hat{p}_{\mu_{j}}(\Delta \cdot \hat{p})^{j} \Delta_{\mu_{j+1}} \cdots \Delta_{\mu_{n}}=c_{\phi} \Delta_{\mu_{1}} \cdots \Delta_{\mu_{n}}
$$

where $\hat{p}_{\mu}$ is a unit vector in the unit three sphere, $S^{3}$, in Euclidean momentum space. The removal of trace terms can be accomplished, heuristically, by taking $\Delta_{\mu}$ to be null. The angular averaging over $S^{3}$ that is left is a type of integral explained in [12]. The result of doing this integral is

$$
\frac{i g_{Y M}^{2} C_{2}(R)}{16 \pi^{2}} \sum_{j=0}^{n-1} \frac{2^{j} j !}{(2 j+2) ! !} \Delta_{\mu_{1}} \cdots \Delta_{\mu_{n}}=c_{\phi} \Delta_{\mu_{1}} \cdots \Delta_{\mu_{n}}
$$

where $n ! !=\prod_{j=0}^{\lfloor n / 2\rfloor}(n-2 j)$. Simplifying, we obtain

$$
c_{\phi}=\frac{i g_{Y M}^{2} C_{2}(R)}{32 \pi^{2}} \sum_{j=1}^{n} \frac{1}{j} \sim \frac{i g_{Y M}^{2} C_{2}(R)}{32 \pi^{2}} \ln n
$$

for large $n$. This shows that the wavefunction renormalization, and hence the anomalous dimension, do scale as $\lambda \ln n$ for large $n$, as desired. ${ }^{4}$ Calculations of this sort serve as classic demonstrations of logarithmic scaling violation in deep inelastic scattering. It is fascinating to see logarithms also in the supergravity dual, which is far from the perturbative regime! Of course, here the scaling is $\sqrt{\lambda} \ln n$. The turnover from $O(\lambda)$ to $O(\sqrt{\lambda})$ behavior is reminiscent of the coefficient for Wilson loops $[11,18,17]$. For consistency with our supergravity result, the $k$-loop correction to the anomalous dimension should grow for large $S$ as $\lambda^{k} \ln S$. This behavior is indeed observed in both supersymmetric and non-supersymmetric gauge theories [16]. The successes [18, 17] in computing circular Wilson loops to all orders in the 't Hooft coupling lead us to hope that similar exact resummations (perhaps of rainbow graphs) might lead to a direct comparison between strongly coupled field theory and string theory near the leading edge of the Regge trajectory. This line of thought is particularly attractive in light of the fact that the states we are considering are nearly back-to-back Wilson loops - compare figure 1b) to 1c).

We should emphasize that the conclusion that in $\mathcal{N}=4 \mathrm{SYM}$ the $k$-loop correction to the anomalous dimension grows at most as $\ln S$ is highly non-trivial. In gauge theories individual graphs at $k$-loop order grow as fast as $\lambda^{k}(\ln S)^{2 k-1}$ [21]. Often there are cancellations, however. ${ }^{5}$ Analyses of the two-loop [19, 20], and all higher

\footnotetext{
${ }^{4}$ We thank T. Petkou for pointing out to us that a similar result has been obtained in [15].

${ }^{5}$ We thank E. Witten for pointing this out to us.
} 
order corrections [16] indeed indicate that the anomalous dimensions grow only as $\ln S$ perturbatively, and probably also non-perturbatively. Remarkably, there seems to be little difference in the behavior of these anomalous dimensions between the $\mathcal{N}=4$ SYM theory and QCD. This should have an interesting effect on the behavior of the deep inelastic scattering.

We should also note that there exist simple bilocal operators whose expansion generates all higher spin operators introduced above:

$$
\begin{gathered}
\operatorname{Tr}\left\{\phi^{*}(x) \operatorname{Pexp}\left(i \int_{x}^{y} A_{\mu} d x^{\mu}\right) \phi(y) \operatorname{Pexp}\left(-i \int_{x}^{y} A_{\mu} d x^{\mu}\right)\right\} \\
=\sum_{n=0}^{\infty} \mathcal{O}_{\mu_{1} \ldots \mu_{n}}^{\phi}(x-y)^{\mu_{1}} \cdots(x-y)^{\mu_{n}} .
\end{gathered}
$$

Thus, if the separation between $x$ and $y$ is taken to be light-like then expanding in powers of $(x-y)^{\mu}$ we recover precisely the higher spin operators made out of two scalar fields and some number of covariant derivatives that we have been studying. Similar bilocal operators can be made by replacing $\phi$ by a fermion field or $F_{\mu \nu}$. The growth of the anomalous dimensions with $S$ is clearly related to the presence of the path-ordered exponentials in (57), [16]. It is also tempting to think that the "folded string" nature of the bilocal operators is related to the spinning string picture of the dual states in $\mathrm{AdS}_{5}$.

\section{Discussion}

Our discussion of spinning string solitons has so far been phrased in terms of the global $\mathrm{AdS}_{5}$ metric of Lorentzian signature. If we continue to Euclidean signature, both in spacetime and on the world sheet, then what we have done can be rephrased in terms of the Euclidean $A d S_{5}$ space written in Poincaré coordinates.

Introducing coordinate $r=\sinh \rho$ in $(2)$, we may write the Euclidean continuation of the metric as

$$
d s^{2}=\left(1+r^{2}\right) d t^{2}+\frac{d r^{2}}{1+r^{2}}+r^{2} d \Omega_{3}^{2}
$$

This may be brought to the form

$$
d s^{2}=d \varphi^{2}+e^{2 \varphi} \sum_{i=1}^{4} d x_{i}^{2}=d \varphi^{2}+e^{2 \varphi}\left(d y^{2}+y^{2} d \Omega_{3}^{2}\right)
$$

by the coordinate change

$$
\varphi=t+\frac{1}{2} \ln \left(1+r^{2}\right), \quad y=\frac{r}{\sqrt{1+r^{2}}} e^{-t} .
$$


Now we may substitute the Euclidean continuation of the spinning string soliton studied in section 3 to derive a soliton in Poincaré coordinates.

It is of further interest to consider more general metrics of the form

$$
d s^{2}=d \varphi^{2}+a^{2}(\varphi) \sum_{i=1}^{4} d x_{i}^{2}
$$

which are relevant to more general gauge theories. In this case finding a spinning string soliton solution in closed form is less straightforward. If we attempt to transform to $S^{3}$ slicing of the geometry, then we generally arrive at a time-dependent metric of the form

$$
f(r, t) d t^{2}+g(r, t) d r^{2}+r^{2} d \Omega_{3}^{2} .
$$

The time-dependence in these coordinates is a sign of broken conformal invariance. Suppose, however, that in the UV (for large $\varphi$ ) the metric is asymptotic to $\mathrm{AdS}_{5}$ : $a(\varphi) \rightarrow e^{\varphi}$. Then in the $S^{3}$ slicing of the geometry the metric approaches (58) for large $r$. For a spinning string with very large spin $S$, the dominant contributions to conformal dimension and spin arise from this large $r$ region where the metric is approximately $\mathrm{AdS}_{5}$. We conclude, therefore, that the relation (3) applies in the limit of large $S$ for all gauge theories that approach the $\mathcal{N}=4$ SYM theory asymptotically in the UV. We hope to return to construction and study of solitons in theories with different UV asymptotics in a future publication.

In this paper we have shown that some characteristically stringy effects can be extracted from the AdS/CFT duality by studying states whose Lorentz spin $S$ is much greater than $R^{2} / \alpha^{\prime}=\sqrt{\lambda}$. The dual description of gauge theory operators with such large $S$ cannot be given in terms of local fields in $\mathrm{AdS}_{5}$; instead it involves strings stretched across $\mathrm{AdS}_{5}$ in such a way that they probe the metric near its boundary. Our results are reliable for $R^{2} / \alpha^{\prime} \gg 1$, but one could hope that they are applicable qualitatively even for $R^{2} / \alpha^{\prime}$ of order 1 .

In fact, recently there appeared interesting ideas on how to extend the AdS/CFT duality to large $N$ gauge theory at weak 't Hooft coupling $\lambda[22,23,7,24,25,26]$. In this case the relation between $R^{2} / \alpha^{\prime}$ and $\lambda$ is not known precisely. Naively, $R^{2} / \alpha^{\prime}$ is of order $\lambda$ in the weak coupling limit, but it is also possible that it approaches a number of order 1 (there might not be an unambiguous definition of $R$ when it becomes as small as $\sqrt{\alpha^{\prime}}$ ). If we suppose that our results may be extrapolated to this situation, then the dual description of states with $S \gg 1$ is not in terms of local fields in $A d S_{5}$ but rather in terms of non-local stretched string states. This comment could be relevant to the search for infinite number of higher spin symmetries at $\lambda=0$ because the gauge theory operators we are discussing are precisely the higher spin currents. 
Our results suggest that extra conservation laws, obvious at $\lambda=0$, persist at nonzero coupling in some asymptotic sense. Since we have $(\Delta-S) / S \ll 1$ for operators on the leading Regge trajectory, we find that the spacing of dimensions of these operators is asymptotically integral. These facts are not enough to predict an exact conservation law, as one may be able to do if $\Delta=S+2$. But they are suggestive of asymptotic conservation laws as $S$ tends to $\infty$.

\section{Acknowledgments}

We are very grateful to J. Maldacena for collaboration at early stages of this project. We also thank B. Altshuler, M. Bertolini, K. Efetov, G. Korchemsky, P. Ouyang and E. Witten for useful discussions. The work of SSG is supported in part by DOE grant DE-FG02-91ER40671 and an Outstanding Junior Investigator award. The work of IRK and AMP is supported in part by the NSF Grant PHY-9802484.

\section{References}

[1] A.M. Polyakov, "String theory and quark confinement," Nucl. Phys. B (Proc. Suppl.) 68 (1998) 1, hep-th/9711002; "The wall of the cave," hep-th/9809057.

[2] J. Maldacena, "The large N limit of superconformal field theories and supergravity," Adv. Theor. Math. Phys. 2 (1998) 231-252, hep-th/9711200.

[3] S. S. Gubser, I. R. Klebanov, and A. M. Polyakov, "Gauge theory correlators from non-critical string theory," Phys. Lett. B428 (1998) 105-114, hep-th/9802109.

[4] E. Witten, "Anti-de Sitter space and holography," Adv. Theor. Math. Phys. 2 (1998) 253-291, hep-th/9802150.

[5] I.R. Klebanov, "World volume approach to absorption by nondilatonic branes," Nucl. Phys. B496 (1997) 231, hep-th/9702076; S.S. Gubser and I.R. Klebanov, "Absorption by branes and Schwinger terms in the world volume theory," Phys. Lett. B413 (1997) 41, hep-th/9708005.

[6] N. Berkovits, "Super-Poincare covariant quantization of the superstring," JHEP 0004 (2000) 018, hep-th/0001035.

[7] A. M. Polyakov, "Gauge fields and space-time," hep-th/0110196. 
[8] D. Berenstein, J. Maldacena, and H. Nastase, "Strings in flat space and pp waves from $\mathrm{N}=4$ super Yang Mills," hep-th/0202021.

[9] R. R. Metsaev, "Type IIB Green-Schwarz superstring in plane wave Ramond-Ramond background," Nucl. Phys. B625 (2002) 70, hep-th/0112044.

[10] M. Blau, J. Figueroa-O'Farrill, C. Hull and G. Papadopoulos, "Penrose limits and maximal supersymmetry," hep-th/0201081; "A new maximally supersymmetric background of IIB superstring theory," JHEP 0201 (2002) 047, hep-th/0110242.

[11] J. Maldacena, "Wilson loops in large N field theories," Phys. Rev. Lett. 80 (1998) 4859, hep-th/9803002; S. J. Rey and J. Yee, "Macroscopic strings as heavy quarks in large N gauge theory and anti-de Sitter supergravity," Eur. Phys. J. C 22 (2001) 379, hep-th/9803001.

[12] D. J. Gross and F. Wilczek, "Asymptotically free gauge theories. 2," Phys. Rev. D9 (1974) 980-993; H. Georgi and D. Politzer, "Electroproduction Scaling in an Asymptotically Free Theory of Strong Interactions," Phys. Rev. D9 (1974) 416-420.

[13] O. Aharony, S. S. Gubser, J. Maldacena, H. Ooguri, and Y. Oz, "Large N Field Theories, String Theory And Gravity," Phys. Rept. 323 (2000) 183-386, hep-th/9905111.

[14] H.J. de Vega and I.L. Egusquiza, "Planetoid String Solutions in $3+1$ Axisymmetric Spacetimes," Phys. Rev. D54 (1996) 7513 [hep-th/9607056].

[15] F. A. Dolan and H. Osborn, "Superconformal symmetry, correlation functions and the operator product expansion," hep-th/0112251.

[16] G.P. Korchemsky, "Asymptotics of the Altarelli-Parisi- Lipatov Evolution Kernels of Parton Distributions," Mod.Phys.Lett. A4 (1989) 1257-1276; G.P. Korchemsky, G. Marchesini, "Structure Function for Large $\mathrm{x}$ and Renormalization of Wilson Loop," Nucl.Phys. B406 (1993) 225-258, hep-ph/9210281.

[17] N. Drukker and D. J. Gross, "An exact prediction of N $=4$ SUSYM theory for string theory," J. Math. Phys. 42 (2001) 2896-2914, hep-th/0010274.

[18] J. K. Erickson, G. W. Semenoff, and K. Zarembo, "Wilson loops in N =4 supersymmetric Yang-Mills theory," Nucl. Phys. B582 (2000) 155-175, hep-th/0003055. 
[19] E.G. Floratos, D.A. Ross, and C.T. Sachrajda, Nucl. Phys. B152 (1979) 493.

[20] A. Gonzalez-Arroyo and C. Lopez, Nucl. Phys. B166 (1980) 429.

[21] D. Gross, Lectures at Les Houches 1975.

[22] M.A. Vasiliev, "Higher Spin Gauge Theories: Star Product and AdS Space," hep-th/9910096.

[23] P. Haggi-Mani and B. Sundborg, "Free Large N Supersymmetric Yang-Mills Theory as a String Theory," hep-th/0002189; B. Sundborg, "Stringy Gravity, Interacting Tensionless Strings and Massless Higher Spins," hep-th/0103247.

[24] E. Witten, Talk at the John Schwarz 60-th Birthday Symposium, http://theory.caltech.edu/jhs60/witten/1.html.

[25] E. Sezgin and P. Sundell, "Doubletons and 5D Higher Spin Gauge Theory," hep-th/0105001.

[26] A. Mikhailov, "Notes On Higher Spin Symmetries," hep-th/0201019. 